\title{
Síndrome de fragilidade no idoso: uma revisão narrativa
}

\author{
The frailty syndrome in elderly: a narrative review
}

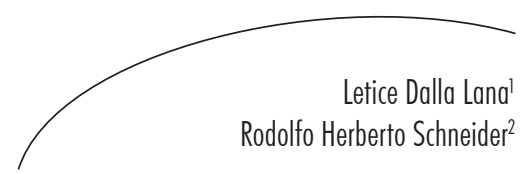

\section{Resumo}

Este estudo se propôs a realizar uma revisão narrativa sobre a temática da síndrome da fragilidade no idoso, visto que a literatura ainda carece de unanimidade quanto a sua conceituação. A revisão foi realizada nos meses de maio e junho de 2012, por meio dos descritores frailty, aged, frail elderly e aging e seus respectivos correspondentes em português nas bases de dados LILACS, MEDLINE, SciELO e PubMed. A amostra contemplou artigos publicados de 1990 a 2012, resultantes de pesquisas primárias quantitativas, qualitativas e estudos teóricos. Os 58 artigos selecionados na amostra final apontaram diferentes instrumentos metodológicos para identificar e quantificar a presença de fragilidade nos idosos. Demonstraram que a fragilidade no idoso envolve múltiplas dimensões e pode ser influenciada por fatores ligados ao próprio indivíduo ou fatores externos, os quais resultam em diferentes conceitos da síndrome da fragilidade no idoso. Conclui-se que os pesquisadores da área estão investindo na geração de conhecimento com o intuito de elaborar uma única definição que possa contemplar com mais propriedade o conceito da síndrome de fragilidade no idoso, contribuindo na mudança de desfechos adversos, podendo intervir na prevenção e promoção de saúde nesses idosos.

\section{Abstract}

This study aimed to conduct a literature review on the topic of the frailty syndrome in the elderly, since the literature still lacks unanimity on its conceptualization. One review was held in May and June 2012, through the descriptors frailty, aged, frail elderly and aging and their counterparts in Portuguese in LILACS, MEDLINE, PubMed and SciELO databases. The sample included articles published from 1990 to 2012, resulting from quantitative, qualitative primary research and theoretical studies. The 58 articles selected in the final sample showed different methodological tools to identify and quantify the presence of frailty in the elderly. They showed that frailty in elderly involves multiple dimensions and can be influenced by factors related to the individual

Palavras-chave: Idoso. Idoso Fragilizado. Envelhecimento. Fragilidade.
Key words: Elderly. Frail Elderly. Aging. Fragility.

\footnotetext{
Programa de Pós-graduação em Gerontologia Biomédica. Pontifícia Universidade Católica do Rio Grande do Sul. Porto Alegre, RS, Brasil.

2 Instituto de Geriatria, Pró-reitoria de Pesquisa e Pós-graduação. Pontifícia Universidade Católica do Rio Grande do Sul. Porto Alegre, RS, Brasil.
} 
himself or external factors, which result in different concepts of the frailty syndrome in the elderly. We conclude that researchers in the field are investing in the generation of knowledge in order to develop a single definition that can include more properly the concept of frailty syndrome in the elderly, helping change adverse outcomes, and intervening in the prevention and promotion health to them.

\section{INTRODUÇÃO}

A crescente expectativa de vida da população tem demonstrado aumento significativo no número de idosos, o que se reflete nas condições de saúde, morbidade e limitações funcionais. ${ }^{1}$ Segundo o Instituto Brasileiro de Geografia e Estatística (IBGE), o Brasil está em processo de mudança, considerando-se o acelerado crescimento no envelhecimento populacional, a redução na taxa de fecundidade e a reestruturação da pirâmide etária. ${ }^{2}$ Tal mudança está gerando grandes desafios para a sociedade, que precisa ser capaz de promover o envelhecimento saudável e ativo, bem como viabilizar recursos sociais e econômicos em todo o mundo. ${ }^{3,4}$

O envelhecimento pode ser entendido como um processo dinâmico e progressivo, em que há modificações morfológicas, funcionais, bioquímicas e psicológicas, com perda progressiva da capacidade de adaptação do indivíduo ao meio ambiente, assim como maior prevalência de processos patológicos que demonstram maior incapacidade com as inúmeras perdas, incluindo papel social, renda, posição social, independência e estrutura anatômica. ${ }^{5,6}$ Desta forma, o envelhecimento leva a uma maior vulnerabilidade a fatores internos e externos, que predispõem ao risco de morbimortalidade.

Nesse contexto, pode ocorrer o desenvolvimento da fragilidade no idoso, caracterizada como uma síndrome clínica cujos sinais e sintomas são preditores de diversas complicações futuras em sua saúde, o que torna esta condição um importante problema de saúde pública. ${ }^{7,8}$

Estudos afirmam que a fragilidade no idoso caracteriza-se pela presença de sinais e sintomas como perda de peso não intencional (5kg nos últimos cinco anos), autorrelato de fadiga, diminuição da força de preensão, redução das atividades físicas, diminuição na velocidade da marcha (lentidão) e diminuição das relações sociais. ${ }^{7-9}$ Demonstram que a fragilidade abrange os domínios físico, psicológico e social, como afirmado por Gobbens et al. ${ }^{10}$ A forma de rastreamento e identificação da predisposição da presença de fragilidade pode ser mensurada por diferentes métodos. Contudo, a falta de profissionais habilitados e a dificuldade de uma avaliação ampliada da saúde, rompendo a fragmentação do cuidado, indicam a necessidade de mais estudos com idosos frágeis. ${ }^{11}$

A literatura em Geriatria e Gerontologia tem mostrado a importância da síndrome da fragilidade entre os idosos, pois impulsiona o risco de quedas, incapacidade, hospitalização e morte. ${ }^{11}$ Deste modo, o presente estudo se propôs a realizar uma revisão narrativa sobre a temática da síndrome da fragilidade no idoso.

\section{METODOLOGIA}

Para realizar este levantamento bibliográfico foram consultadas, nos meses de maio e junho de 2012, referências sobre a temática da síndrome da fragilidade no idoso, nas bases de dados LILACS, MEDLINE, SciELO e PubMed. Adicionalmente, foram consultados cinco livros de Geriatria e Gerontologia disponibilizados na Biblioteca Irmão José Ótão, da Pontifícia Universidade Católica do Rio Grande do Sul (PUCRS), além do banco de dissertações e teses da CAPES. Foram utilizados os seguintes descritores: frailty, aged, frail elderly e aging e seus respectivos correspondentes em português. Também foram acessadas as bases de dados do IBGE. Na seleção inicial foram 
identificados, no período de 1990 a 2012, 648 artigos, seis teses e oito dissertações, resultantes de pesquisas primárias quantitativas, qualitativas e estudos teóricos.

Como critério de inclusão, definiu-se o período de publicação de 22 anos pela possibilidade de inclusão de um número maior de artigos, visto que se constatou, em busca preliminar, que o tema "fragilidade no idoso" ainda tem sido pouco estudado. Além disso, incluíram-se artigos disponibilizados na íntegra na língua portuguesa e inglesa, dissertações e teses na língua portuguesa, e revisão sistemática ou metanálise de todos os ensaios clínicos randomizados controlados, ensaios clínicos controlados sem randomização, estudos de casos-controle e estudos de coorte publicados em revistas. Os critérios de exclusão adotados foram os estudos de revisão sistemáticos descritivos e qualitativos, opinião de autoridades, relatórios de comitês de especialistas e editoriais. Na leitura dos resumos, com base nos critérios de elegibilidade adotados pelo estudo, foram excluído 413 artigos, duas teses e quatro dissertações. Após a leitura do texto completo, foram excluídos 177 artigos, totalizando uma amostra final de 58 artigos, quatro teses e quatro dissertações que foram analisadas na íntegra.

\section{RESULTADOS E DISCUSSÃO}

O termo "fragilidade" teve início em meados de 1980, quando Whoodhouse ${ }^{12}$ propôs o conceito de fragilidade nos indivíduos com 65 anos de idade ou mais e que dependiam de outras pessoas para poderem realizar as atividades cotidianas. Desta forma, os estudos associavam a fragilidade à perda da autonomia e dependência para realizar as atividades básicas de vida diária (ABVD) ${ }^{13-17}$

Conforme afirmou Carvalho Filho, ${ }^{5}$ Brocklehurst criou um modelo de fragilidade que contemplava os componentes biológico, social e psicológico no ano de 1982. A partir dessa abordagem, identificou-se que a fragilidade pode se manifestar em qualquer faixa etária, podendo estar presente não apenas na velhice. ${ }^{17,18}$ No entanto, quando associada ao envelhecimento, tem sido amplamente aceita como uma síndrome clínica, de natureza multifatorial, caracterizada por um estado de vulnerabilidade fisiológica resultante da diminuição das reservas de energia e da habilidade de manter ou recuperar a homeostase após um evento desestabilizante. ${ }^{18,19}$ Nessa linha, Teixeira ${ }^{20}$ mostrou que o conceito de fragilidade seria unidimensional, visto que agrega a vulnerabilidade e baixa resposta aos fatores de estresse, bem como maior suscetibilidade a doenças e à presença de síndromes geradoras de incapacidade. ${ }^{20-22}$

Contudo, observa-se que o termo "fragilidade" ainda não apresenta uma definição consensual entre os pesquisadores, pois alguns autores identificam o constructo multidimensional da fragilidade. ${ }^{23-25}$ Este constructo multiprofissional salienta a etiologia da síndrome dependente da história de vida do indivíduo, pois pode ser modificado diante dos fatores biológicos, psicológicos e sociais, resultando em déficits individuais em cada contexto particular. ${ }^{20,24,25}$

Além disso, outros estudos afirmam que o início e o curso da síndrome da fragilidade são afetados não somente por fatores pessoais de natureza cognitiva, psicológica, espiritual, sociais e físicos, mas também por fatores ambientais de ordem financeira, interpessoal, legal e institucional, que culminam em maior risco de desfechos clínicos adversos, dentre os quais o declínio funcional, quedas, hospitalização, institucionalização e morte.,26-29 No entanto, pode ser evitada ou postergada quando identificada precocemente com base em seus indicadores, pois esta síndrome leva à deterioração da qualidade de vida, aumento da sobrecarga dos cuidadores e altos custos com cuidados à saúde. ${ }^{29-31}$ Para tanto, o conceito de fragilidade pode ser compreendido como uma síndrome de múltiplas dimensões e que envolve diferentes definições. ${ }^{31}$

A definição mais utilizada sobre a síndrome da fragilidade é que ela representa uma síndrome biológica caracterizada por diminuição da 
reserva homeostática e redução da capacidade do organismo de resistir às intempéries, resultando em declínios cumulativos em múltiplos sistemas fisiológicos, causando vulnerabilidade e efeitos adversos. ${ }^{19}$ Assim, o resultado cumulativo desses declínios em múltiplos sistemas do corpo humano poderia constituir a base do que é frequentemente considerado o fenótipo da fragilidade: a vulnerabilidade aos fatores estressores e o comprometimento da capacidade de manter a homeostase, bem como a incapacidade, dependência parcial ou total em atividades básicas da vida diária (ABVD), da maior presença de comorbidades ou de idosos mais debilitados e vulneráveis. ${ }^{19,32,33}$ Neste contexto e juntamente com outros autores, a fragilidade é identificada como uma síndrome associada à idade e caracterizada por manifestações como perda de peso e massa muscular, diminuição da força de preensão, fadiga, instabilidade postural e redução da ingestão de alimentos, aumentando o risco para uma evolução desfavorável diante das agressões externas e das doenças agudas. ${ }^{12,19,33-35}$

Como não há consenso sobre sua definição, existem diferentes instrumentos utilizados pelos profissionais da saúde para identificar e quantificar a presença da síndrome da fragilidade no idoso, tanto no âmbito da pesquisa como na prática clínica, para avaliar os declínios decorrentes em idosos.

Observa-se que o fenótipo da fragilidade é um método de identificação e mensuração da fragilidade nos idosos que utiliza, como critérios integrantes da síndrome, a perda de peso não intencional (pelos menos $5 \mathrm{Kg}$ no último ano), exaustão avaliada por autorrelato de fadiga, diminuição da força de preensão da mão dominante, baixo nível de atividade física e baixa velocidade ao caminhar (lentidão). ${ }^{19}$

Ainda dentro dos critérios componentes da síndrome da fragilidade, outros estudos ${ }^{19,36-40}$ têm mostrado marcadores adicionais ao fenótipo proposto por Fried et al. ${ }^{11}$ para a mensuração da fragilidade em idosos, com a inclusão da Escala de Katz, que avalia as atividades básicas da vida diária (ABVD); a Escala de Lawton, para as atividades instrumentais da vida diária (AIVD); a Escala Internacional de Eficácia de Quedas; o Miniexame do Estado Mental; e a Medida de Independência Funcional, que avalia o desempenho e a capacidade funcional de idosos. ${ }^{19,36-40}$

Um fato a ser considerado, entretanto, é que as diferentes escalas não enfocam a temática em questão, a fragilidade do idoso, como é demonstrada na Escala de Fragilidade de Edmonton (EFE), visto que a fragilidade pode deteriorar a qualidade de vida do idoso. Para tal, sabe-se que independentemente do conceito ideal de qualidade de vida, esta se constitui pela busca constante do bem-estar de forma indissociável das condições do modo de viver, como: saúde, moradia, educação, lazer, transporte, liberdade, trabalho, autoestima, entre outras.

A EFE é considerada um instrumento mais complexo, pois é constituída de nove domínios que evidenciam, com maior nitidez, a fragilidade no idoso, que são: cognição, estado geral de saúde, independência funcional, suporte social, uso de medicamentos, nutrição, humor, continência e desempenho funcional. ${ }^{41}$

A EFE foi traduzida e validada para utilização no Brasil em 2008, onde foi aplicada em uma amostra de 137 idosos residentes de uma comunidade no município de Ribeirão Preto-SP. ${ }^{42}$ De acordo com o estudo, 16,4\% dos entrevistados apresentaram fragilidade leve; $9 \%$, fragilidade moderada; e 4,5\% apresentaram fragilidade severa. Juntamente com a aplicação da escala, outros dados foram levantados por meio de instrumentos que avaliaram o perfil sociodemográfico, cognitivo e a independência funcional dos idosos. Os resultados identificaram que a maior prevalência de fragilidade está entre os idosos mais velhos, ou seja, acima de 80 anos, que apresentam déficit cognitivo e do sexo feminino. ${ }^{16,41}$

Em estudo com idosos de uma comunidade norte-americana, os autores associaram positivamente sexo feminino, etnia afroamericana, situação socioeconômica mais 
precária, baixo nível de escolaridade, pior estado de saúde, morbidades preexistentes caracterizadas como doenças crônicas não transmissíveis (DCNT) e incapacidades à maior presença da síndrome. A síndrome da fragilidade para esse estudo identificou prevalência de fragilidade de $6,9 \% .{ }^{19}$ Outros estudos desenvolvidos em dez países europeus, envolvendo idosos de outros centros internacionais, identificaram prevalência de fragilidade que oscila de $7 \%$ a $17 \%$, sendo os de menor prevalência os idosos comunitários acima de 65 anos, e os de maior prevalência os idosos com fenótipo para a fragilidade. ${ }^{42-44}$

Em uma comunidade de idosos de Belo Horizonte, foi encontrada prevalência de $13,2 \%$, em que os indivíduos frágeis estavam situados num grupo etário mais velho, com menor renda e nível educacional, viúvos, com maior número de morbidades, maior dependência na ABVD e AIVD e com pior autopercepção de saúde. ${ }^{45}$ Remor et al..$^{36}$ identificaram, em um ambulatório de gerontologia de uma capital brasileira, 31\% de idosos frágeis, $53 \%$ pré-frágeis e $16 \%$ não frágeis. Nesse estudo, a fragilidade foi significativamente associada ao tipo de moradia, perda de peso não intencional, fadiga e fraqueza autorreferidas, inatividade, capacidade funcional diminuída, consumo de bebida alcoólica e internações hospitalares nos últimos anos. Já outro estudo desenvolvido em uma comunidade da cidade de São Paulo, que avaliou a síndrome da fragilidade em idosos por meio da EFE, identificou 39,1\% de idosos frágeis, sendo 18,3\% com fragilidade leve, $11,3 \%$ com fragilidade moderada e 9,6\% com fragilidade severa. ${ }^{22}$

Além da maior prevalência de fragilidade constatada em estudos brasileiros, outras características associadas têm sido observadas e poderiam explicar esses achados. Marin et al., ${ }^{46}$ em estudo com idosos de uma Unidade de Saúde da Família (USF) de São Paulo, constataram que 61,8\% são mulheres, 68,1\% são analfabetos ou têm o primeiro grau incompleto, $36,9 \%$ vivem sem o companheiro, $72,4 \%$ são considerados pobres ou muito pobres e 161 (53,5\%) vivem apenas da aposentadoria. Já outro estudo realizado com idosas inscritas em um Programa de Saúde da Família de Macéio-AL, identificou que 48,5\% viviam com menos de um salário mínimo percapita, apresentavam uma a duas morbidades, $12,7 \%$ apresentaram dependência parcial para a AIVD e 50,7\% eram sedentárias ou insuficientemente ativas. ${ }^{47}$ Outro estudo com idosos vinculados à Estratégia da Saúde da Família (ESF) do município de Uberaba-MG identificou uma prevalência de fragilidade de 19,9\%, sendo 19,7\% para homens e $20 \%$ para as mulheres. ${ }^{48}$

Adicionalmente, estudo conduzido por Castro Borges et $a .^{49}$ identificou que os profissionais da ESF prestam cuidado domiciliar ao idoso dependente quando o mesmo já se encontra num estágio mais avançado de comprometimento da capacidade funcional. Desta forma, as orientações em saúde são efetuadas quando alguns sinais de declínio já estão presentes, principalmente pelo fato de $56 \%$ dos idosos entrevistados durante a visita domiciliar já apresentarem mobilidade física prejudicada (acamados).

A limitação encontrada nesse estudo se refere à metodologia adotada pelos estudos analisados, pois sem um instrumento único e padronizado para mensurar a fragilidade no idoso não se pode realizar uma comparação entre os dados. Inferese, assim, a necessidade de implementar um método unificado de rastreamento e identificação da fragilidade do idoso.

\section{CONCLUSÃO}

Com base nesta revisão, verifica-se que diferentes autores estão investindo na geração de conhecimento, no sentido de elaborar uma definição que possa contemplar com mais propriedade o conceito da síndrome de fragilidade no idoso, bem como suas características mais prevalentes.

Os dados identificam que o perfil dos idosos frágeis pode estar associado a etnia, tipo de instrumento, local do estudo e tamanho da amostra, evidenciando abrangência na prevalência de idosos frágeis no âmbito da comunidade, 
hospital e instituições de longa permanência para idosos. Contudo, os estudos mostram que os idosos de maior idade, com menor escolaridade, doença crônica prévia, institucionalizados, em uso contínuo de medicações, com queda da própria altura nos últimos anos e poucas relações sociais estão presentes entre os idosos considerados frágeis.

Pela importância do tema e pela necessidade de modificar desfechos adversos ocasionados pela presença da síndrome da fragilidade, os autores concluem que uma padronização

\section{REFERÊNCIAS}

1. Parahyba MI, Simões CCS. Disability prevalence among the elderly in Brazil. Ciênc Saúde Coletiva 2006;11(4):967-74.

2. Instituto Brasileiro de Geografia e Estatística. Pesquisa Nacional por Amostra de Domicílios (PNAD): sinopse do Censo Demográfico 2010. Rio de Janeiro: IBGE; 2011.

3. Gordilho A, Nascimento JS, Ramos LR, Freire MPA, Espindola N, Maia R, et al. Desafios a serem enfrentados no terceiro milênio pelo setor saúde na atenção integral ao idoso. Rio de Janeiro: UnATI/ UERJ; 2000.

4. Organização Mundial da Saúde, Organização PanAmericana de Saúde. Envelhecimento ativo: uma política de saúde. Brasília,DF:OMS; 2005.

5. De Carvalho Filho ET. Fisiologia do envelhecimento. In: Papaléo Netto M. Gerontologia: a velhice e o envelhecimento em visão globalizada. São Paulo: Atheneu; 2005. p. 60-70.

6. Eliopoulos C. Enfermagem Gerontológica. $5^{\mathrm{a}}$ ed. Porto Alegre: Artmed; 2005. p. 74-91.

7. Linck CL, Crossetti MGO. Fragilidade no idoso: o que vem sendo produzido pela enfermagem. Rev Gaúcha Enferm 2011;32(2):385-93.

8. Bergman H, Ferruci L, Guralnik J, Hogan DB, Hummel S, Karunananthan S, et al. Frailty: an emerging research and clinical paradigm: issues and controversies. J Gerontol 2007;62(7):731-7.

9. Fabricio SCC, Rodrigues RAP. Revisão da literatura sobre síndrome da fragilidade e sua relação com o envelhecimento. Rev RENE 2008;9(2):113-9. conceitual deve ser atingida, objetivando a elaboração de condutas voltadas a prevenção e promoção de saúde nesses idosos, visto que a síndrome de fragilidade é complexa e demanda ações isoladas para prevenir, retardar ou impedir sua progressão no envelhecimento.

\section{AGRADECIMENTO}

À Pontifícia Universidade Católica do Rio Grande do Sul-PUCRS, pela concessão de bolsa de mestrado a Letice Dalla Lana, pelo ProBolsa.
10. Gobbens RJJ, Van Assen MALM, Luijkx KG, Wijnen-Sponselee MTh, Schols JMGA. The Tilburg Frailty Indicator: psychometric properties. J Am Med Dir Assoc 2010;11(5):344-55.

11. Fried LP, Ferrucci L, Darer J, Williamson JD, Anderson G. Untangling the concepts of disability, frailty and comorbidity: implications for improved targeting and a care. J Gerontol Ser A Biol Sci Med Sci 2004;59(3):255-63.

12. Woodhouse KW. Frailty and ageing. Age Ageing 1997;26:245-46.

13. Gillick M. Pinning down frailty. J Gerontol Ser A Biol Sci Med Sci 2001;56(3):M134-35.

14. Morley J, Perry H, Miller D. Something about frailty [Editorial]. J Gerontol 2002;57(11):M698-709.

15. Morley J. Developing novel therapeutic approaches to frailty. Curr Pharm Des 2009;15(1):3384-95.

16. Fabricio-Wehbe SCC. Adaptação cultural e validação da "Edmonton Frail Scale". Definições de fragilidade em idosos: uma abordagem multiprofissional [tese]. São Paulo: Universidade de São Paulo, Escola de Enfermagem de Ribeirão Preto; 2008.

17. Fulop T, Larbi A, Witkowski JM, McElhaney J, Loeb M, et al. Aging, frailty and age-related diseases. Biogerontology 2010;11(5):547-63.

18. Walston J, Hadley EC, Ferrucci L, Guralnik JM, Newman AB, Studenski SA, et al. Research agenda for frailty in older adults: toward a better understanding of physiology and etiology: summary from the american geriatrics society/national institute on aging research conference on frailty in older adults. J Am Geriatr Soc 2006;54(6):991-1001. 
19. Fried L, Tangen CM, Walston J, Newman AB, Hirsch C, Gottdiener J, et al. Frailty in Older Adults: evidence for a phenotype. J Gerontol 2001;56(3):M146-56.

20. Teixeira IN. Definições de fragilidade em idosos: uma abordagem multiprofissional [dissertação]. São Paulo: Universidade Estadual de Campinas, Faculdade de Educação; 2006.

21. Carvalho Neto N. Envelhecimento bem sucedido e envelhecimento com fragilidade. In: Ramos LR, Toniolo Neto J. Guias de medicina ambulatorial hospitalar da UNIFESP - Escola Paulista de Medicina. São Paulo: Manole; 2005. p. 9-25.

22. Fhon JRS. A prevalência de quedas em idosos e a sua relação com a fragilidade e a capacidade funcional [tese]. Ribeirão Preto: Escola de Enfermagem de Ribeirão Preto; 2011.

23. Maciel AC, Guerra RO. Influência dos fatores biopsicossociais sobre a capacidade funcional de idosos residentes no nordeste do Brasil. Rev Bras Epidemiol 2007;10(2):178-89.

24. Bergman H, Beland F, Karunananthan S, Hummel S, Hogan D, Wolfson C. Developpement d'un cadre de travail pour comprendre et étudier la fragilité. Gérontol soc 2004;109(1):15-29.

25. Ben-Shlomo Y, Kuh D. A life course approach to chronic disease epidemiology: conceptual models, empirical challenges and interdisciplinary perspectives. Int J Epidemiol 2003;31(1):285-93.

26. Rockwood K, Hogan D, Macknigth C. Conceptualisation and measurement of frailty in elderly people. Drugs Aging 2000;17(4):295-302.

27. Rolfson DB, Majumdar SR, Tsuyuki RT, Tahir A, Rockwood K. Validity and reliability of the Edmonton Frail Scale. Age Ageing 2006;35(5):526-9.

28. Teixeira INDO, Neri AL. A Fragilidade no Envelhecimento: fenômeno multidimensional, multideterminado e evolutivo. In: De Freitas EV, Py Ligia, editoras. Tratado de Geriatria e Gerontologia. 2a ed. Rio de Janeiro: Guanabara Koogan; 2006. p. 32-77.

29. Hardy SE, Dubin JA, Holford TR, Gill TM. Transitions between states of disability and independence among older persons. Am J Epidemiol 2005;161(6):575-84.

30. Gill TM, Baker DI, Gottschalk M, Peduzzi PN, Allore $\mathrm{H}$, Byers A. A program to prevent functional decline in physically frail, elderly persons who live at home. N Engl J Med 2002;347(14):1068-74.

31. Duarte YAO. Indicadores de fragilidade em pessoas idosas, visando o estabelecimento de medidas preventivas. Bol Inst Saúde. São Paulo. 2009;47:49-52.
32. Rockwood K, Howlett SE, Mackinigth C. Prevalence, attributes, and outcomes of fitness and frailty in community-dwelling older adults: report from the canadian study of health and aging. J Gerontol 2004;59(12):10-29.

33. Santos EGS. Perfil de fragilidade em idosos comunitários de Belo Horizonte: um estudo transversal [dissertação]. Minas Gerais: Universidade Federal de Minas Gerais; 2008.

34. Costa EFA. Idoso frágil. In: Hargreaves LHH. Geriatria. Brasília, DF: SEEP; 2006. p.171-8.

35. Macedo C, Gazzola JM, Najas M. Síndrome da fragilidade no idoso: importância da fisioterapia. Arq Bras Ciências da Saúde 2008;33(3):177-84.

36. Remor CB, Bós AJG, Werlang MC. Características relacionadas ao perfil de fragilidade no idoso. Sci Med 2011;21(3):107-12.

37. Kawasaki K, Diogo M. Impacto da hospitalização na independência funcional do idoso em tratamento clínico. Acta Fisiátrica 2005;12(2):55-60.

38. Silva SLA, Vieira RA, Arantes P, Dias RC. Avaliação de fragilidade funcionalidade e medo de cair em idosos atendidos em um serviço ambulatorial de geriatria e gerontologia. Fisioter Pesqui 2009;16(2):125-29.

39. Farias CA. Fragilidade e desempenho cognitivo em idosos clientes de uma operadora de saúde da zona norte da cidade do Rio de Janeiro [dissertação]. Rio de Janeiro: UERJ; 2011.

40. Maciel ACC, Guerra RO. Influência dos fatores biopsicossociais sobre a capacidade funcional de idosos residentes no nordeste do Brasil. Rev Bras Epidemiol 2007;10(1):178-89.

41. Fabricio-Wehbe SCC, Schiaveto FV, Vendrusculo TRP, Haas VJ, Dantas RAS, Rodrigues RAP. Adaptação cultural e validade da Edmonton Frail Scale - EFS em uma amostra de idosos brasileiros. Rev Latinoam Enferm 2009;17(6):1-7.

42. Cesari M, Leeuwenburgh C, Lauretani F, Onder G, Bandinelli S, Maraldi C et al. Frailty syndrome and skeletal muscle: results from the Invecchiare in Chianti study. Am J Clin Nutr 2006;83(5):1142-8.

43. Ávila-Funes JA, Amieva H, Barberger-Gateau P, Le Goff M, Raoux N, Ritchie K, et al. Cognitive impairment improves the predictive validity of the pheotype of frailty for adverse health outcomes: the three-city study. J Am Geriatr Soc 2009,57(3):453-61.

44. Santos-Eggimann B, Cuénoud P, Spagnoli J, Junod $\mathrm{J}$. Prevalence of frailty in middle-aged and older community-dwelling europeans living in 10 countries. J Gerontol Ser A Biol Sci Med Sci 2009;64(6):675-81. 
45. Santos EGS. Perfil de fragilidade em idosos comunitários de Belo Horizonte: um estudo transversal [dissertação]. Belo Horizonte: Universidade Federal de Minas Gerais; 2008.

46. Marin MJS, Cecílio LCO. Necessidades de saúde de idosos de uma Unidade de Saúde da Família. Rev Bras Geriatr Gerontol 2009;12(1):63-76

47. Santos AB, Ribeiro AS. Dados sociodemográficos e condições de saúde de idosas inscritas no PSF de Maceió, AL. Rev Bras Geriatr Gerontol 2011;4(4):613-23.
48. Tribess S. Estudo da associação entre o índice de fragilidade e variáveis demográficas de saúde e comportamentais em idosos [tese]. Brasília, DF: Universidade de Brasilia, Programa de Pós-Graduação em Ciências da Saúde; 2012.

49. Borges MMMC, Telles JL. O cuidado do idoso no contexto familiar: percepção da equipe de saúde da família. Rev Bras Geriatr Gerontol 2010;13(3):349-60.

Recebido: 22/10/2012

Revisado: 05/11/2013

Aprovado: 09/6/2014 